\title{
Distribuição temporal do ermitão Clibanarius vittatus (Anomura, Diogenidae) no litoral do Paraná
}

\author{
Sara R. Sampaio, Setuko Masunari \& Kirstern L. F. Haseyama
}

Programa de Pós-graduação em Zoologia, Departamento de Zoologia, Centro Politécnico, Universidade Federal do Paraná, Bairro Jardim das Américas, Caixa Postal 19020, 81.531-990 Curitiba, PR, Brasil. (sararsp@yahoo.com.br; setmas@ufpr.br)

\begin{abstract}
Temporal distribution of the hermit crab Clibanarius vittatus (Anomura, Diogenidae) from Paraná State coast, Brazil. A study on the temporal fluctuation of the population structure of the hermit crab Clibanarius vittatus (Bosc, 1802) was carried

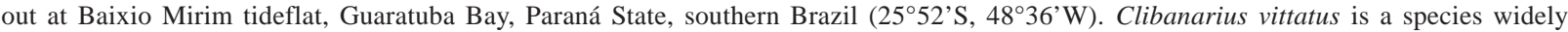
studied, but studies of population biology of these hermit crabs in different regions are scarce. Samplings were done in the intertidal zone, monthly, from April/2005 to March/2006. A total of 1187 hermit crabs were obtained, among which, 949 males, 22 intersexes, 204 nonovigerous females and 12 ovigerous females. The cephalothoracic shield length (CSL) was measured and grouped in 10 size classes. Males were distributed in 10 CSL classes while intersexes and females occurred only in six size classes. Males predominated in every month and in the whole population. All demographic categories were present all year round, except intersexes and ovigerous females. These were uncommon and occurred from late spring until autumn and in august/2005. The reproduction was considered seasonal-continuous, with higher intensity in the warmer months. The observed sex ratio in this population, that was different from other ones, corroborates the theory of migration for this species. The present research constitutes the first record of reproductive period of $C$. vittatus population occurring in warm subtropical shallow waters.
\end{abstract}

KEYWORDS. Intertidal hermit crabs, reproductive period, intersexes, sex ratio, size classes.

RESUMO. Um estudo sobre a flutuação temporal da estrutura populacional do ermitão Clibanarius vittatus (Bosc, 1802) foi realizado no

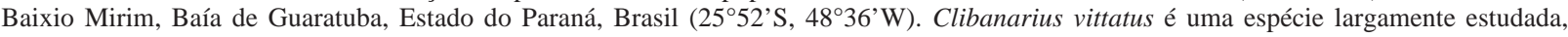
entretanto estudos da biologia populacional desta em diferentes regiões são escassos. Coletas mensais foram realizadas na zona entremarés, de abril/2005 a março/2006. Foram obtidos 1187 ermitões, dos quais, 949 machos, 22 indivíduos intersexo, 204 fêmeas não ovígeras e 12 fêmeas ovígeras. O comprimento do escudo cefalotorácico (CEC) foi medido e agrupado em 10 classes de tamanho. Machos ocorreram em 10 classes de CEC, enquanto fêmeas e intersexos em apenas seis. A razão de sexos total e mensal foi a favor dos machos. Todas as categorias demográficas estiveram presentes o ano inteiro, com exceção dos intersexos e das fêmeas ovígeras. Estas foram pouco frequentes e ocorreram do final da primavera até o outono, e em agosto de 2005. O período reprodutivo foi considerado contínuosazonal, com maior intensidade nos meses mais quentes. A razão de sexos nesta população, que é diferente das demais estudadas, corrobora a teoria de migração proposta para esta espécie. O presente estudo constitui o primeiro registro do período reprodutivo desta espécie em águas rasas de clima subtropical quente.

PALAVRAS-CHAVE. Ermitões da zona entremarés, período reprodutivo, intersexos, razão de sexos, classes de tamanho.

Clibanarius vittatus (Bosc, 1802) é um ermitão com ampla distribuição geográfica no Atlântico Ocidental, do leste dos Estados Unidos até as Antilhas, incluindo o Golfo do México, e da Venezuela até o sul do Brasil, no Estado de Santa Catarina (Melo, 1999). Ele foi considerado resistente às mudanças extremas na temperatura, salinidade e nível da água no litoral do Golfo do México (EUA) (Fotheringham, 1975).

A estrutura populacional desta espécie de ermitão é conhecida principalmente no sul da América do Norte (FotHERINGHAM, 1975; LOWERY \& NELSON, 1988) e no litoral do Estado de São Paulo (Negreiros-Fransozo et al., 1991; Turra \& Leite, 2000; SAnt'Anna et al., 2006). Da América do Norte, são conhecidos aspectos da biologia de $C$. vittatus como a migração sazonal para as águas mais profundas, um comportamento efetivo para proteger estes animais das temperaturas muito frias das águas de superfície (Fotheringham, 1975), e segregação sexual causada por esta migração diferencial entre os sexos (LOWERY \& NELSON, 1988). Entretanto, para as populações do litoral brasileiro, estas migrações ainda não foram descritas.

A reprodução desta espécie é sazonal e ocorre de abril a outubro no hemisfério norte (FothERINGHAM, 1975; LOWERY \& NeLSON, 1988) e de outubro a julho no litoral de São Paulo (TurRa \& Leite, 2000).

Clibanarius vittatus tem sido intensamente estudado desde a segunda metade do século passado (HAZLETt, 1968a, b; Young, 1978, 1979), entretanto, dados sobre a biologia de diferentes populações são escassos, o que dificulta a comparação de populações provenientes de diferentes regiões e o estabelecimento de um padrão para esta espécie de ermitão.

Sobre populações de $C$. vittatus ocorrentes no litoral do Paraná, não existem estudos até o momento. O presente estudo descreve as flutuações sazonais na estrutura da população de ermitões no Baixio Mirim no que diz respeito aos aspectos do tamanho dos indivíduos, da razão de sexos e do período reprodutivo.

\section{MATERIAL E MÉTODOS}

Os ermitões foram coletados manualmente no Baixio Mirim ( $\left.25^{\circ} 52^{\prime} \mathrm{S}, 48^{\circ} 36^{\prime} \mathrm{W}\right)$, Baía de Guaratuba, PR, durante as marés baixas de sizígia, mensalmente, de abril de 2005 a março de 2006. Cada amostra mensal consistiu de esforço 
de procura de 100 ermitões, procurando abranger indivíduos de todos os tamanhos, para não subamostrar os menores.

No laboratório, os ermitões foram retirados de suas conchas, identificados, tiveram o sexo reconhecido e foram medidos no comprimento do escudo cefalotorácico (CEC) com paquímetro digital (0,01 mm de precisão). Eles foram agrupados em 10 classes de tamanho com amplitude de $1 \mathrm{~mm}$ de CEC cada uma. Os intersexos foram excluídos do cálculo da razão sexual.

A normalidade dos dados de CEC foi testada com o teste de Kolmogorov-Smirnov $(\mathrm{P}<0,05)$. Para comparar a distribuição do CEC de machos, fêmeas e intersexos foi utilizado o teste não paramétrico de Mann-Whitney, visto que estes dados não apresentaram distribuição normal, já na comparação entre fêmeas e fêmeas ovígeras utilizouse o teste t-student, pois ambos os grupos acusaram normalidade dos dados. Para estimativa da razão sexual foi aplicado o teste Qui-quadrado $(\mathrm{GL}=1)$. A correlação entre temperatura e número de fêmeas ovígeras foi realizada por postos de Spearman, utilizando-se o teste $\mathrm{t}$ para verificação da existência de correlação. Para todos os testes foi aceito o intervalo de significância de 0,95 e o valor exato de $\mathrm{P}$ foi citado sempre que fornecido pelos programas estatísticos utilizados (STATistica 7.0; GRAPHPAD INSTAT 3.0).

Os dados de temperatura média mensal do ar foram cedidos pelo Instituto Tecnológico SIMEPAR, do Centro Politécnico da UFPR.

\section{RESULTADOS}

Foram coletados 1187 ermitões $C$. vittatus dos quais, $949(79,9 \%)$ machos, $22(1,9 \%)$ intersexos, 204 (17,2\%) fêmeas não ovígeras e $12(1,0 \%)$ fêmeas ovígeras. A média do CEC dos machos foi de $6,13 \pm 1,31 \mathrm{~mm}$, com uma amplitude de 2,97 a 11,56 mm. Os intersexos apresentaram uma amplitude do CEC de 3,76 a 9,45 mm e

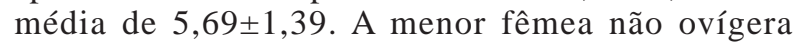
apresentou CEC de 2,97 $\mathrm{mm}$ e a maior de 8,24 $\mathrm{mm}$, e a média de 5,00 $\pm 0,82 \mathrm{~mm}$. O CEC das fêmeas ovígeras foi de 3,95 $\mathrm{mm}$ na menor, e 7,75 $\mathrm{mm}$ na maior, com média de

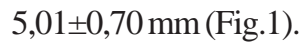

A população apresentou dimorfismo sexual quanto ao tamanho: fêmeas atingiram médias do CEC sempre menores do que os machos $(\mathrm{P}<0,0001)$ ou intersexos $(\mathrm{P}<0,0001)$. Os indivíduos intersexo apresentaram média do CEC significativamente igual a dos machos (MannWhitney, $\mathrm{P}=0,8738$ ), assim como as fêmeas ovígeras e não-ovígeras não apresentam diferença significativa nesta dimensão (teste t, $\mathrm{P}=0,5273$ ) (Fig. 1).

Os machos foram mais abundantes na população em todos os meses do período de estudo, seguidos das fêmeas e dos intersexos (Fig. 2). A razão de sexos variou de 2,81:1,0 (M:F) em março/06 a 12,9:1,0 em abril/05. As fêmeas ovígeras são tão raras quanto os indivíduos intersexo, estas ocorreram em sete enquanto os intersexos ocorreram em oito dos 12 meses amostrados. Em contraste, machos e fêmeas não-ovígeras estiveram presentes ao longo de todo o período (Fig. 3).

Os machos ocorreram em 10 classes de CEC e as fêmeas e os indivíduos intersexo em apenas seis. $\mathrm{Na}$ maioria dos meses, a classe modal dos machos foi a que compreende indivíduos com CEC de 5,00 a 5,99 mm enquanto que a das fêmeas, com indivíduos de CEC entre 4,00 e 4,99 mm. Fêmeas ovígeras não ocorreram na classe que compreende os menores indivíduos (CEC de 2 a 2,99 $\mathrm{mm}$ ) e a partir da classe formada pelas maiores fêmeas (CEC de 8 a 8,99 mm) (Fig. 3). A razão sexual na maioria das classes esteve a favor dos machos, entretanto, na classe modal das fêmeas a razão sexual foi significativamente semelhante a 1:1 em todos os meses (teste Qui quadrado, GL=1, P<0,05), com exceção de abril e dezembro. As demais classes diferiram significativamente no número de machos e fêmeas ou não apresentaram número de indivíduos suficiente para análise estatística $(\mathrm{n}<5)$.

As fêmeas ovígeras foram pouco frequentes e ocorreram em abril/05, agosto/05 e de novembro/05 a março/06 (Figs. 3, 4). A temperatura média mensal do ar nos meses de coleta variou de $16,7^{\circ} \mathrm{C}$ (setembro/05) a $25,2^{\circ} \mathrm{C}$ (janeiro/06), a mínima mensal de $13,9^{\circ} \mathrm{C}$ (julho/05) a $24,8^{\circ} \mathrm{C}$ (janeiro/06) e a máxima mensal, de $19,5^{\circ} \mathrm{C}$ (setembro/05) a $25,8^{\circ} \mathrm{C}$ (abril/05). Fêmeas ovígeras ocorreram em meses com temperatura média acima de $18^{\circ} \mathrm{C}$, e apresentaram correlação com a temperatura média mensal do ar ( $r=0,58 ; t=2,24 ; \mathrm{P}=0,049)$ (Fig. 4).

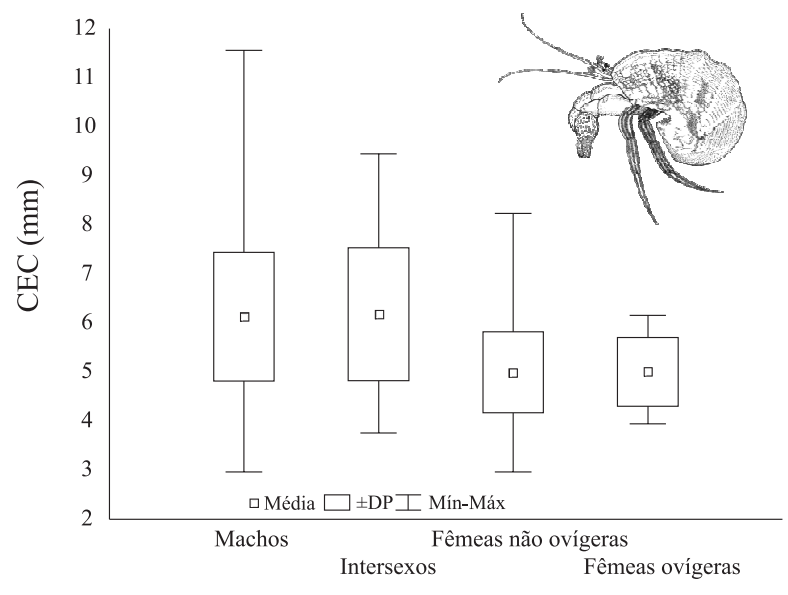

Figura 1. Média, desvio padrão (DP) e amplitude (MínimoMáximo) do comprimento do escudo cefalotorácico (CEC) das categorias demográficas de Clibanarius vittatus (Bosc, 1802) coletados no período de abril de 2005 a março de 2006 no Baixio Mirim, Baía de Guaratuba, PR.

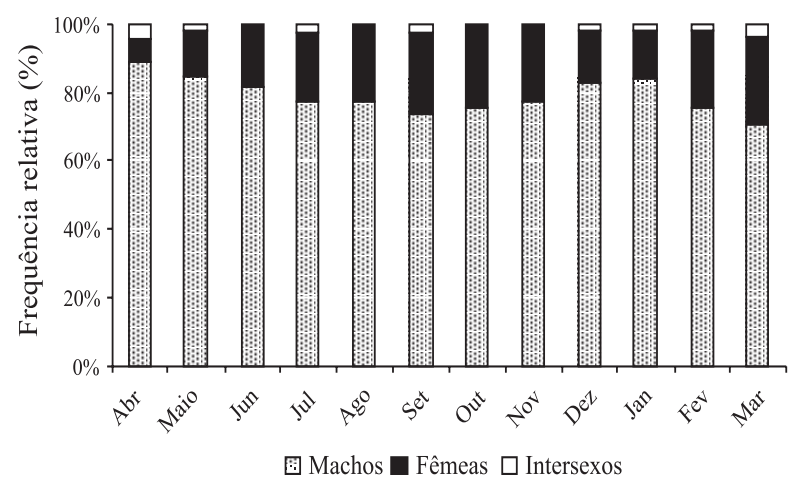

Figura 2. Flutuação da frequência relativa das categorias demográficas de Clibanarius vittatus (Bosc, 1802) coletados no período de abril de 2005 a março de 2006 no Baixio Mirim, Baía de Guaratuba, PR. 


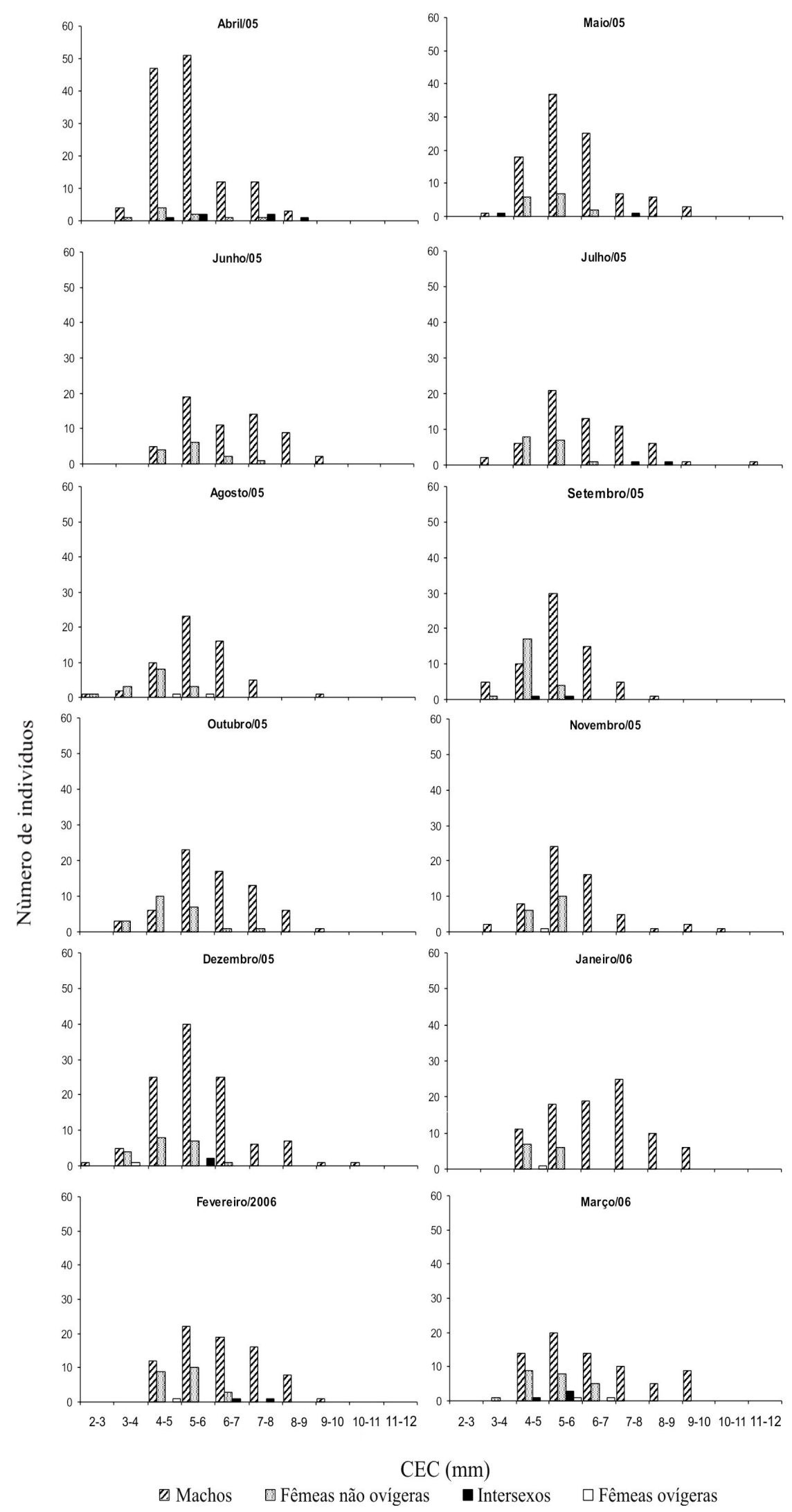

Figura 3. Distribuição da frequência absoluta das categorias demográficas nas diferentes classes de comprimento do escudo cefalotorácico (CEC) de Clibanarius vittatus (Bosc, 1802) coletados no período de abril de 2005 a março de 2006 no Baixio Mirim, Baía de Guaratuba, PR. 


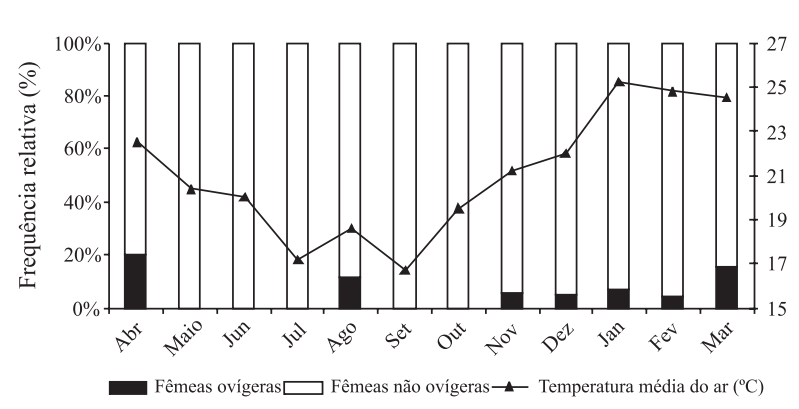

Figura 4. Flutuação da temperatura média mensal do ar $\left({ }^{\circ} \mathrm{C}\right)$ e da frequência relativa de fêmeas não ovígeras e ovígeras de Clibanarius vittatus (Bosc, 1802) coletados no período de abril de 2005 a março de 2006 no Baixio Mirim, Baía de Guaratuba, PR.

\section{DISCUSSÃO}

O tamanho médio dos indivíduos da população estudada (CEC $=5,86 \pm 1,32 \mathrm{~mm})$ diferiu muito pouco da estudada no litoral de São Paulo (CEC = 6,98 $\pm 1,04 \mathrm{~mm}$ ) (TurRa \& Leite, 2000). As fêmeas possuem tamanho médio menor (CEC fêmeas não ovígeras $=5,00 \pm 0,82 \mathrm{~mm}$ e CEC fêmeas ovígeras $=5,01 \pm 0,70)$ do que as estudadas no litoral paulista (CEC fêmeas não ovígeras $=6,76 \pm 0,70$ $\mathrm{mm}$ e CEC fêmeas ovígeras $=6,47 \pm 0,79)$. Além disso, os intersexos têm CEC médio semelhante ao dos machos, diferentemente do encontrado por TURRA \& LEITE (2000), os quais registram um valor intermediário ( $\mathrm{CEC}=7,25 \pm 0,73$ $\mathrm{mm}$ ) entre machos e fêmeas. Estas diferenças nos valores podem estar relacionadas com o tamanho diferente de amostra nestes estudos ou ainda com taxas diferentes de crescimento nas populações em função das variáveis abióticas.

O dimorfismo sexual em tamanho é comum dentre vários gêneros de ermitões (TurRa \& Leite, 2000; Benvenuto \& Gherardi, 2001; Litulo, 2005; Mantelatto et al., 2007; Ayres-Peres \& Mantelatto, 2008), e parece ser característica de C. vittatus, pois tanto nas populações do Hemisfério Norte (Fotheringham, 1975; Lowery \& Nelson, 1988) como nas do Sul (Negreiros-Fransozo et al., 1991; TURRA \& LeITE, 2000) os machos estão presentes nas maiores classes de tamanho e, sua classe modal é sempre maior do que a das fêmeas. O dimorfismo sexual nas populações de ermitões pode ser explicado pelo fato de que machos possuem mais energia disponível para o crescimento (não gastam energia para produzir ovos), pela ação da seleção sexual (machos maiores obtêm vantagem na competição por uma parceira) ou pela competição intra-específica pela ocupação das conchas (Bertness, 1981; Hartnoll, 1982). O fato de fêmeas ovígeras estarem ausentes na maior classe de tamanho pode estar relacionado com a senescência reprodutiva (SOMERS, 1991).

A razão de sexos de $C$. vittatus observada no Baixio Mirim diferiu muito das demais populações estudadas, enquanto neste local houve predominância de machos, em outros estudos, as fêmeas foram mais abundantes (Fotheringham, 1975; Lowery \& Nelson, 1988; Negreiros-Fransozo et al., 1991; TuRra \& Leite, 2000). A razão de sexos diferente de 1:1 é comum entre os crustáceos e pode ser causada por reversão sexual, migração diferencial, mortalidade diferencial e diferentes taxas de crescimento (WENNER, 1972).

Quanto à variação mensal da razão de sexos, a população estudada manteve o predomínio de machos em todas as classes de tamanho na maioria dos meses, com a ausência de fêmeas em algumas classes e a razão de 1:1 na classe modal das fêmeas. Em contraste, estudos realizados com $C$. vittatus em outros estados mostraram que a razão de sexos tem predominância de fêmeas sempre, mas alcança a razão de 1:1 em determinados meses (LOWERY \& Nelson, 1988; TuRRa \& Leite, 2000). Baseado nessas diferenças de razão sexual em diferentes classes de tamanho, Fotheringham (1975) inferiu uma migração sazonal e diferencial entre machos e fêmeas no litoral norte-americano, onde machos grandes deixam a zona intertidal e migram para as águas profundas no começo do verão e, em seguida, as fêmeas menores o fazem no final do outono. Essa migração seria uma medida adaptativa de proteção contra as baixas temperaturas prevalentes durante o inverno nas águas intertidais.

A razão de sexos com predominância de machos pode indicar uma possível migração diferencial dos sexos, como proposta por FotHERINGHAM (1975) e corroborada por LOWERY \& Nelson (1988). Assim, fêmeas adultas permaneceriam no baixio somente porque os machos se encontram em maior abundância para reprodução, e que, depois de fecundadas estas fêmeas migrariam para uma região mais estável e provavelmente com maior salinidade, já que este parece ser um fator importante para o desenvolvimento das larvas (Fotheringham \& BAGNALL, 1976). A frequência extremamente baixa das fêmeas ovígeras no Baixio Mirim, quando comparada com a encontrada por TURRA \& LEITE (2000) no litoral paulista, sustenta esta inferência. Neste estado, as fêmeas ovígeras perfazem mais de $50 \%$ nos meses de pico reprodutivo, e elas possuem uma média menor no tamanho quando comparadas com as do Baixio Mirim.

A baixa frequência de intersexos é uma característica das populações de $C$. vittatus (TuRRa \& Leite, 2000) e o papel destes é de difícil interpretação em todas as espécies de ermitões em que estes são encontrados (GuZEv \& ZавотіN, 2007). Inicialmente, os intersexos eram considerados machos funcionais por apresentarem a capacidade de fecundar uma fêmea (TURRA, 2004), entretanto, a descoberta de um indivíduo intersexo ovado demonstrou que o mesmo pode ser também uma fêmea funcional (TURRA, 2007).

O período reprodutivo de ermitões é fortemente relacionado à sazonalidade das variáveis abióticas e à história evolutiva da espécie no local e não à proximidade taxonômica e geográfica das espécies (TURRA \& LeITE, 2000). Apesar do número de fêmeas ovígeras do presente estudo apresentar correlação com a temperatura média mensal, não é possível afirmar que a não captura destas represente a ausência de reprodução, já que a ausência das fêmeas nos demais meses pode ter sido apenas um artifício de amostragem. Com base nesta premissa, o período reprodutivo registrado do final da primavera até o outono (abril/05 e de novembro a março/06) e em agosto de 2005, sugeriu uma reprodução contínua-sazonal. Interrupções na ocorrência de fêmeas ovígeras nesta espécie já foram observadas no litoral paulista em agosto 
e setembro (Turra \& Leite, 2000), assim como em populações de ermitões congêneres e de outros gêneros, como Clibanarius chapini Schmitt, 1926 e Clibanarius senegalensis Chevreux \& Bouvier, 1898 (AMEYAwAkumfi, 1975) e Calcinus tibicen (Herbst, 1791) de Ubatuba (Fransozo \& Mantelatto, 1998), que apresentam este padrão de interrupção da reprodução em meses de baixas temperaturas. Na América do Norte, o período reprodutivo de $C$. vittatus está concentrado nos meses de primavera, verão e início do outono (Fortheringham, 1975; Lowery \& NELSON, 1988).

As características da população estudada assemelham-se às observadas em outros litorais do país, com exceção da razão de sexos. Isto salienta a necessidade de estudos de migrações diferenciais para áreas subtidais ou para outras intertidais fora do Baixio Mirim e de comparações entre diferentes populações para se estabelecer um padrão para a espécie.

Agradecimentos. Ao SIMEPAR pelos dados de temperatura do ar do município de Guaratuba, PR. Aos Coordenadores do Programa de Pós-graduação em Zoologia UFPR, pela permissão no uso das instalações. Contribuição No. 1728 do Departamento de Zoologia UFPR.

\section{REFERÊNCIAS BIBLIOGRÁFICAS}

Ameyaw-AKUmfi, C. 1975. The breeding of two sympatric species of tropical hermit crabs, Clibanarius chapini and $C$. senegalensis. Marine Biology 29:15-28.

Ayres-Peres, L. \& Mantelatto, F. L. 2008. Análise comparativa da estrutura populacional do ermitão endêmico do Atlântico Ocidental Loxopagurus loxocheles (Decapoda, Anomura) em duas regiões do estado de São Paulo, Brasil. Iheringia, Série Zoologia, 98(1):28-35.

Benvenuto, C. \& Gherardi, F. 2001. Population structure and shell use in the hermit crab, Clibanarius erythropus: a comparison between Mediterranean and Atlantic shores. Journal of the Marine Biological Association of the United Kingdom 81:77-84

Bertness, M. D. 1981. The influence of shell-type on hermit crab growth rate and clutch size (Decapoda, Anomura). Crustaceana 40(2):197-205

FothERINGHAM, N. 1975. Structure of seasonal migrations of the littoral hermit crab Clibanarius vittatus. Journal of Experimental Marine Biology and Ecology 18:47-53.

Fotheringham, N. \& Bagnall, A. 1976. Seasonal variation in the occurrence of planktonic larvae of sympatric hermit crabs. Journal of Experimental Marine Biology and Ecology 21:279-287.

Fransozo, A. \& Mantelatto, F. L. M. 1998. Population structure and reproductive period of the tropical hermit crab Calcinus tibicen ( Decapoda: Diogenidae) in the region of Ubatuba, São Paulo, Brazil. Journal of Crustacean Biology 18(4):738-745.

Graphpad Software, Inc. 1997. Graphpad instat. Versão 3.00. São Diego.
Guzev, O. \& Zabotin, Y. 2007. Observation of intersexuality in land hermit crabs (Anomura: Coenobitidae). Journal of the Marine Biological Association of the United Kingdom 87:533-536.

Hartnoll, R. G. 1982. Growth. In: Abele, L. G. ed. The biology of Crustacea. New York, Academic. v.2. p.111-196.

Hazlett, B. A. 1968a. Size relationships and aggressive behavior in the hermit crab Clibanarius vittatus. Zeitschrift für Tierpsychologie 25:608-614.

1968b. Stimuli involved in the feeding behavior of the hermit crab Clibanarius vittatus (Decapoda, Paguridea). Crustaceana 15(3):305-311.

Litulo, C. 2005. Population structure and reproduction of the hermit crab Dardanus deformis (Anomura: Diogenidae) in the Indian Ocean. Journal of the Marine Biological Association of the United Kingdom 85:883-887.

Lowery, W. A. \& Nelson, W. G. 1988. Population of the hermit crab Clibanarius vittatus (Decapoda: Diogenidae) at Sebastian Inlet, Florida. Journal of Crustacean Biology 8(4):548-556.

Mantelatto, F. L.; Faria, F. C. R.; Iossi, C. R. \& Biagi, R. 2007. Population and reproductive features of the western Atlantic hermit crab Pagurus criniticornis (Anomura, Paguridae) from Anchieta Island, Southeastern Brazil. Iheringia, Série Zoologia, 97(3):314-320.

Melo, G. A. S. 1999. Manual de Identificação dos Crustacea Decapoda do Litoral Brasileiro: Anomura, Thalassinidea, Palinuridea, Astacidea. São Paulo, Plêiade. 551p.

Negreiros-Fransozo, M. L.; Fransozo, A. \& Hebling, N. J. 1991 Estrutura populacional e determinação do tamanho da concha em 4 espécies de ermitões (Crustacea, Decapoda, Anomura) do litoral paulista. Biotemas 4(2):1335-148.

Sant'Anna, B. S.; Zangrande, C. M.; Reigada, A. L. D. \& Pinheiro, M. A. A. 2006. Shell utilization pattern of the hermit crab Clibanarius vittatus (Crustacea, Anomura) in a estuary at São Vicente, State of São Paulo, Brazil. Iheringia, Série Zoologia, 96(2):261-266.

Somers, K. M. 1991. Characterizing size-specific fecundity in crustaceans. In: Wenner, A. \& Kuris, A. eds. Crustacean Egg Production. Rotterdam, Balkema. p. 357-378.

StatSoft, Inc. 2004. Statistica. Versão 7. Tulsa.

TuRRA, A. 2004. Intersexuality in hermit crabs: reproductive role and fate of gonopores in intersex individuals. Journal of the Marine Biological Association of the United Kingdom 84:757-759

2007. Reproductive role of intersex hermit crabs Crustaceana 80(4):491-494.

Turra, A. \& Leite, F. P. P. 2000. Population Biology and growth of three sympatric species of intertidal hermit crabs in southeastern Brazil. Journal of the Marine Biological Association of the United Kingdom 80:1061-1069.

Wenner, A. M. 1972. Sex ratio as a function of size in marine Crustacea. American Naturalist 106:312-350.

Young, A. M. 1978. Dessication tolerances for three hermit crab species Clibanarius vittatus (Bosc), Pagurus pollicaris Say and $P$. longicarpus Say (Decapoda, Anomura) in the North Inlet Stuary, South Carolina, U. S. A. Estuarine and Coastal Marine Science 6:117-122.

1979. Differential utilization of gastropod shells by three hermit crab species in North Inlet, South Carolina, U. S. A Crustaceana, suppl. 5:101-104. 